\title{
Anomaly Based Sensor Nodes with IoT for Environment Sustainability
}

\author{
Sathya Priya $\mathrm{J}^{\mathrm{a}, 1}$, Bharathi $\mathrm{S} \mathrm{L}^{\mathrm{b}}$, Sathyabama A $\mathrm{R}^{\mathrm{c}}$ \\ ${ }^{a}$ Associate Professor, Dept. of IT, Velammal Engineering College \\ ${ }^{b}$ Assistant Professor, Dept. of ECE, Panimalar Engineering College \\ ${ }^{c}$ Assistant Professor, Dept. of IT, Velammal Engineering College
}

\begin{abstract}
The proposed works implements a prototype of detecting harmful gases in a given environment, along with air and water quality monitoring. This device is capable of detecting temperature and LPG leakage in home environment, monitoring water quality in a given area along with warm body detection and methane concentration inside drains. The temperature and LPG leakage is detected with the help of temperature and MQ-6 sensors, the water quality is monitored with the help of $\mathrm{pH}$ and turbidity sensors. The human presence inside the drain is detected with the help of PIR sensor and methane concentration with the help of MQ-4 sensor. The sensor nodes are based on wifi enabled Arduino microcontroller, that receives data from the sensors and converts it into digital data that are transmitted to the webpage. The main concern of this paper is the real time monitoring of various parameters by implementing low-cost sensors that are useful for both home and the society.
\end{abstract}

Keywords. LPG leakage, methane concentration, temperature sensor, MQ-6 sensor, $\mathrm{pH}$ sensor, turbidity sensor, PIR sensor, Arduino microcontroller.

\section{Introduction}

The air quality in this paper is monitored under home environment. In the last few decades an enormous increase in LPG cylinder transport frequency, both on rail and roads, has been observed. Among other chemical substance, hydrocarbons are very hazardous. With lower capacity level itself, it has high flammable capacity. Thus, for safety reasons it is important for real time monitoring of possible leaks. The sensors are incorporated to trigger a notification when a certain leakage of LPG is detected [1]. Further apart from LPG leakage the temperature can be detected with the help of $\operatorname{lm} 35$ temperature sensor. The water quality monitoring is a holistic approach that detects contaminated water in a given area. Clean water is an expensive resource. Hence it is most important to monitor the parameters that affect the water quality. Existing approach has shown partial improvements in monitoring water quality in real time [2].The two major parameters to be checked are $\mathrm{pH}$ and turbidity of the water. $\mathrm{pH}$ shows the state of the water whereas turbidity shows the nature of the water. For water quality monitoring previous researchers collected water from manual sources at different locations and implemented their approach to determine the quality of water under various parameters. But these approaches did not showed any significant improvements in quality monitoring [3]-[7]. This paper provides a low cost sensor based approach to monitor the quality of water with the help $\mathrm{pH}$ and turbidity sensors.

\footnotetext{
${ }^{1}$ Bharathi S L, Assisstant Professor, Department of ECE, Panimalar Engineering College, Chennai; Email:sl.bharathi18@gmail.com
} 
Passive Infrared sensors (PIR) are low cost sensors that are mostly used as human motion detectors. The common use of PIR sensors have been widely described in [8][9]. The use of PIR sensor in this paper is to detect the presence of any living being inside the drains. Drains are left open in many places due to various reasons humans, animals who pass by can easily trip and fall which causes injuries and suffocate them due to the presence of methane inside the drains. Hence a low cost sensor based approach is used to detect humans along with the methane concentration inside the drains.

\section{Literature survey}

The developed gas detection system, which is named as smart detection system is implemented in the areas of natural gas storage is used to detect harmful gases such as $\mathrm{CH} 4$ and $\mathrm{CO} 2$ [13]. It focuses on detecting gases that causes green house effect. It measures both air and water quality. The air quality parameters such as $\mathrm{CH} 4$ and $\mathrm{CO} 2$ are measured with the help of cost effective sensors, while the water quality parameters such as $\mathrm{pH}$ and EC (Electrical Conductivity) are measured with the help of long running, low cost sensors. The sensor nodes are incorporated in Arduino UNO microcontroller. It fetches the data calculated from various sensors and transmits to the Raspberry pi 3. From the Raspberry pi 3 the obtained data can be retrieved and stored in cloud storage unit and thus enables remote accessing of data.

PIR sensors are devices used to detect existence of obstacles/ object involved [18]. Thus these sensors are used to find the presence of human/animal in the provided region. PIR sensors posses' advantages such as low cost-cost effectiveness, deployment friendly-easier implementation and intend output- appropriate results. Normally these sensors are used in smart home environment. Smart home environment detects the unauthorized entry at home and informs the same to the intend user as an alert message through the mobile App. Since PIR sensors act as surveillance and implemented to monitor safety and security, it is also referred as occupancy detectors [22]. The human exposure into the virtual sensitive zones emits IR heat. This IR radiation emits positively polarized sensitive zone and negatively polarized zone. Combination of positive and negative polarized zone results in sinusoidal voltage pattern. These sensors are implemented either in curtain or dome pattern to detect humans. The sensor makes use of machine learning methods such as classification and clustering in terms of K-Nearest Neighbor (KNN), Support Vector Machine (SVM), simple tree, Linear Discriminate Analysis (LDA), and Artificial Neural Network (ANN) to work with real time observed data.

\section{Architecture and Working}

The proposed working model is implemented in Arduino Node MCU microcontroller. The Arduino reads analog signals as input from sensor nodes and these signals get converted into digital data by ADC converter. With the wifi Raspberry pi these digital data sent to the webpage (Cloud) and can be accessed or monitored from anywhere. The work was incorporated with temperature sensor, PIR sensor, MQ4 gas sensor, $\mathrm{pH}$ 
sensor and turbidity sensor. Temperature sensor monitors the deviation in the allowable temperature, PIR sensors detects obstacles or objects by passing infrared rays, MQ4 sensor detects leakage of any gas and thus detects toxic gas emission, $\mathrm{pH}$ sensor measures the hydrogen-ion in the water suspension and turbidity sensors monitors and detects the turbidity level in the water.

\subsection{Microcontrollers}

Arduino NodeMCU is an advanced version of arduino. It is an open-source platform for Internet of Things (IOT) [24]. It is in-built with ESP-8266 wifi enabled module which does not require any external sources for internet connection. It requires 9600 baud for the rate of transmission. It requires a minimum of $+5 \mathrm{~V}$ and a maximum of $+10 \mathrm{~V}$ power supply. We can connect a maximum of eight digital sensors and one analog sensor. In this paper we have made use of four analog sensors and one digital sensor which ultimately requires an external ADC converter [10].

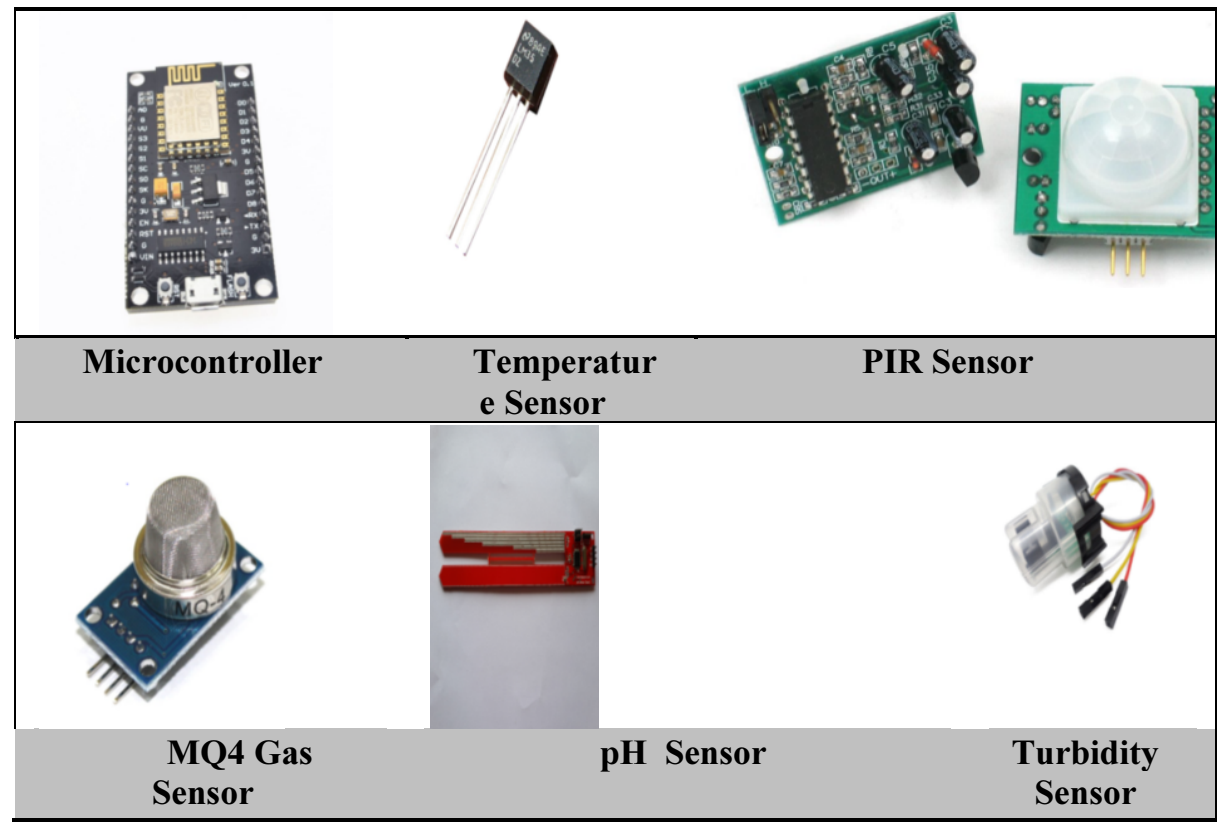

\section{Low cost sensors}

\subsection{Temperature Sensor}

The LM35 is temperature sensor. It is used to measure temperature experienced by it. It is cost effective sensor and readily available for interfacing with microcontroller. It possess some of its properties such as use of only $60 \mu \mathrm{A}$ from its power supply, very low self-heating of $<0.1$ degree centigrade in air. The LM35 is operated over a $-55^{\circ}$ 
to $+150^{\circ} \mathrm{C}$ range [11]. In this paper the $\mathrm{LM} 35$ sensor is used to detect the temperature in air and the resultant data are sent to the webpage where the details are monitored in the form of graph.

\subsection{MQ-6 gas Sensor}

MQ-6 [23] is basically a electrochemical sensor for detecting various gases in the air. It is capable of detecting gases such as LPG, iso-butane, propane, methane and so on. $\mathrm{SnO} 2$ (Tin di-oxide) have lower conductivity in clean air; hence it can be sensitive for MQ-6 sensor [25] to be detected [12].The proposed work is intend to detect LPG and methane gas under home and environment. [13].The MQ-6 sensor is capable of detecting natural gas concentrations of range 100-10000 ppm. The gas sensor module is incorporated to detect the leakage of LPG and presence of methane gas concentration at lower level and notifies the same to the user by triggering or sending message to the mobile phone through the mobile application. The notification is sent to the corresponding mobile number with the help of IoT ThingSpeak Monitor Widget application.

\section{3 pH Sensor}

$\mathrm{pH}$ level is an important parameter in soil, irrigation water and spray tank solutions. In this paper the $\mathrm{pH}$ sensor tests for soil alkalinity / acidity, soil moisture, and sunlight. This sensor provides the nature of water. In traditional methods, $\mathrm{pH}$ sensing was done with the help of conventional $\mathrm{pH}$ electrodes that had various disadvantages [14].

A $\mathrm{pH}$ sensor senses $\mathrm{pH}$ which is a measure of acidity, basicity or alkalinity of water. It is measured based on electrical potential. Pure water is neutral and its $\mathrm{pH}$ should be equal to 7 . The deviation in $\mathrm{pH}$ level tells the acidic and basic nature of water. If the $\mathrm{pH}<7$ is acidic, whereas $\mathrm{pH}>7$ is basic [15].In this paper the $\mathrm{pH}$ sensor values are constantly monitored and are periodically updated to the webpage.

\subsection{Turbidity Sensor}

Turbidity is the most important factor need parameter to be considered to measure the water purity. Turbidity is important parameter to be checked in drinking water [16]. In this proposed work the turbidity sensor is used to check suspended or presence of dust like particles in the water. Turbidity probe and turbidity circuit transmits data to the Arduino microcontroller. When the suspended solid amount in water increases, the water's turbidity level (cloudiness or haziness) increases. It is measured by the optical property of water. Optical property compares the amount of light scattered to the light reflected by from the suspended. As the intensity of scattered light in water is increased, the turbidity level also increases. Turbidity is measured in terms of Nephelometric Turbidity Units (NTU). During low flow, most of the rivers and lakes are pure green or blue in colour hence the turbidity is low, generally lesser than 10 NTU. During the period of high flow, rainstorms, floods, water flows faster and mixes with different particles, which makes the turbidity of water go high.Turbidity probe is made of a plastic covered circuit. The amount of light reflected from water determines the turbidity level of water. As the amount of suspended particles in water increases, 
the amount of light being transmitted decreases. Thus the turbidity level of water can be calculated [17].

\subsection{PIR Sensor}

PIR are widely used as a simple but powerful method to detect human motion. In this paper the PIR sensor is particularly used to detect living being presence inside the drains. They are smaller, less expensive, low-powered, easy to be used and do not wear out easily. Hence they are mostly used in appliances and gadgets used in homes or business. The PIR sensor consists of two partitions, each partition is made up of material that is sensitive to infrared [19][20]. In this paper a topbottom approach is used to mount the sensor inside the drains. Hence when a warm body such a humans or animals passes the sensing range a positive differential change occurs detecting the presence of warm body.

\subsection{MQ4 gas sensor}

This paper focuses on the methane concentration inside drains which are periodically updated to the webpage. Methane gas is mostly produced inside drains that when inhaled by warm bodies can be fatal. Hence it is very important to detect the concentration of these gases as for that person to survive.MQ4 [26] is an easy to use sensor, suitable for sensing methane gas concentrations in the air. It can detect methane gas anywhere from 200 to $10000 \mathrm{ppm}$. It is basically an analog sensor that has a simple drive circuit which is enabled with a power supply of $5 \mathrm{~V}$.

\section{Design}

The working principle of the proposed work is depicted in the below figure. The sensor outputs are collected as analog data and ADC converts the analog to digital data. The results then sent to the Arduino node MCU for analyzing the inputs obtained. Based upon the output obtained trigger can be made to the corresponding registered user.

\subsection{Web Server}

ThingSpeak is an open-source platform. It is based on Internet of Things that is used to store and retrieve data from the sensors that are sent to the internet. It allows the user to collect and store sensor data in the cloud and develop IoT applications. The ThingSpeak IoT platform provides application that helps to analyze and visualize data in real-time. Sensor data inputs can be sent to ThingSpeak from Arduino, RaspberryPi, BeagleBone Black, and other hardware. [21]. 


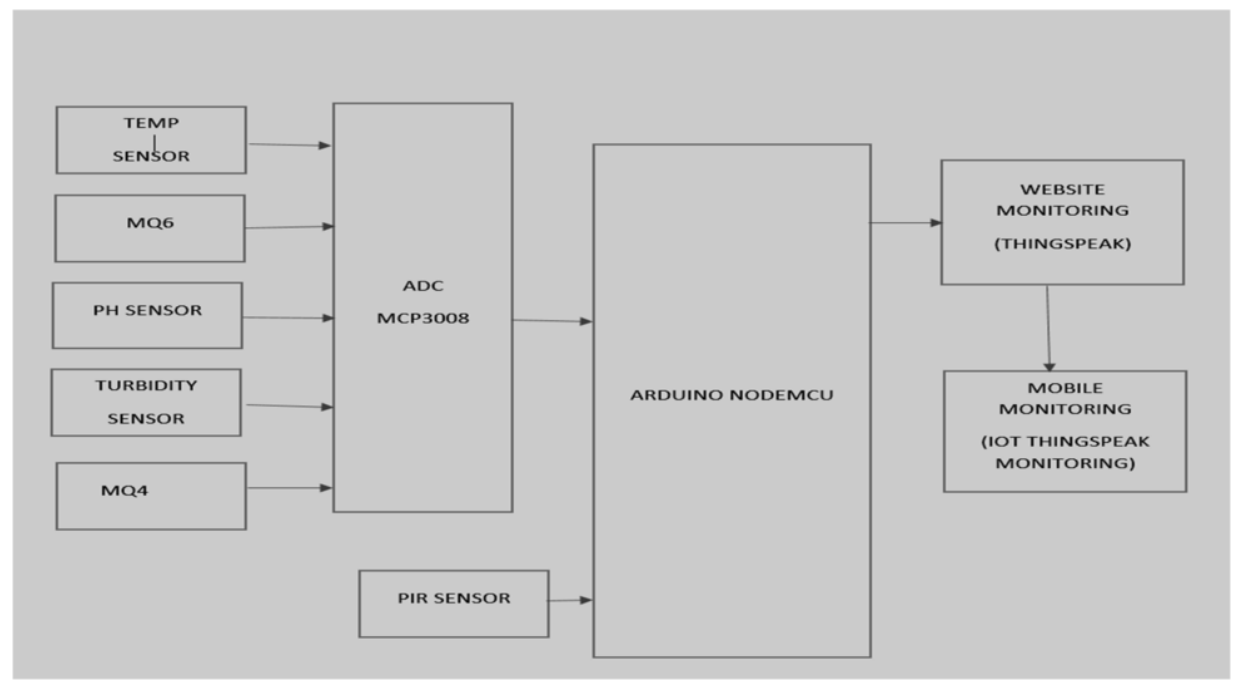

Figure 1. Working Model

\section{Result}

To prove the outcome several experiments were carried out. At this stage each sensors were tested with all possible parameters. The temperature sensor was tested under a hot environment for sufficient result Figure2. The turbidity sensor was tested with both normal water and muddy water Figure3. The gas (MQ-6) sensor was tested with LPG gas and methane gas for the notifications Figure4. The PIR sensor being installed in a top-bottom fashion was tested to detect warm body inside the drains Figure5. Finally the $\mathrm{pH}$ sensor was tested in both pure drinking water and contaminated water for accurate results Figure6.

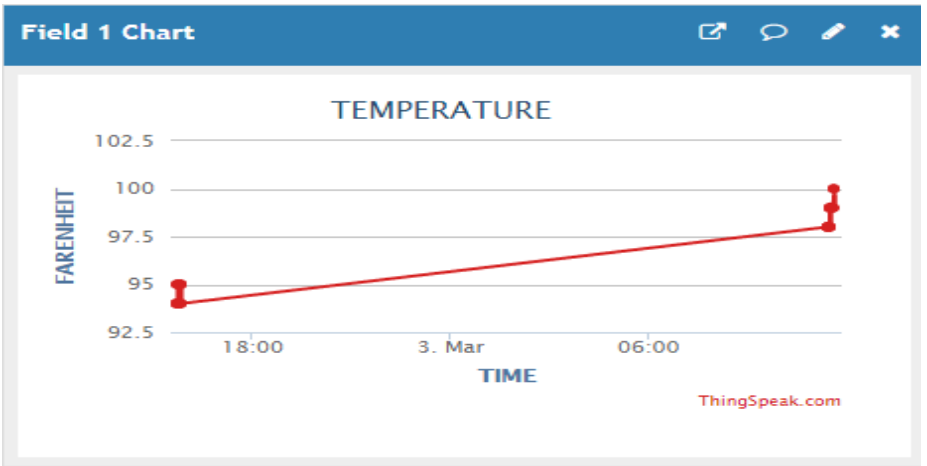

Figure 2. Temperature sensor 


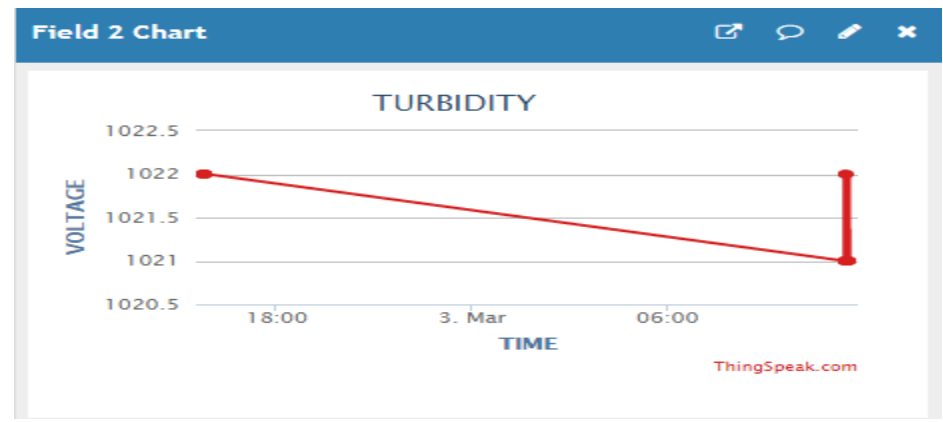

Figure 3. Turbidity sensor

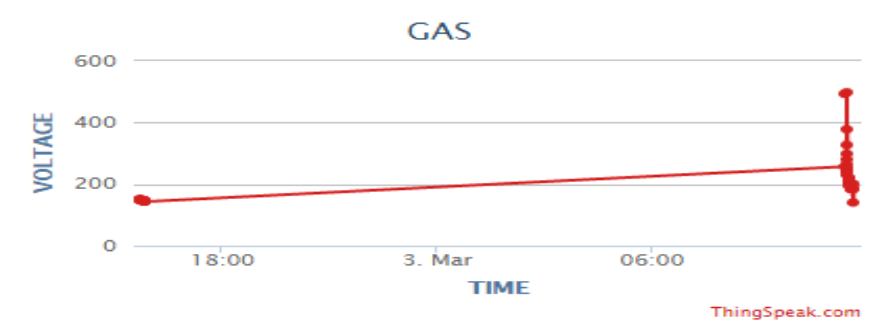

Figure 4. Gas sensor

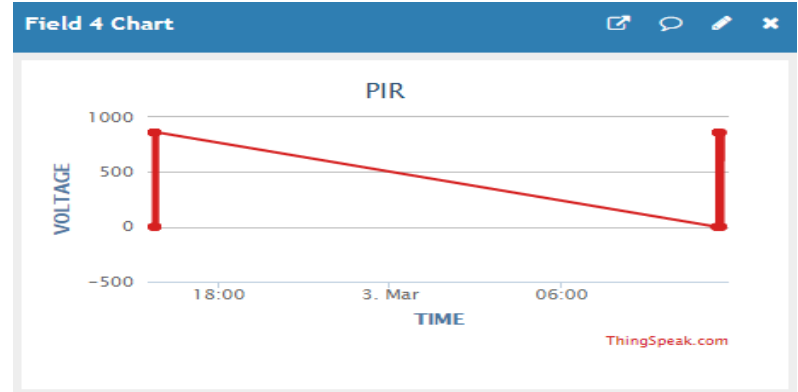

Figure 5. PIR sensor

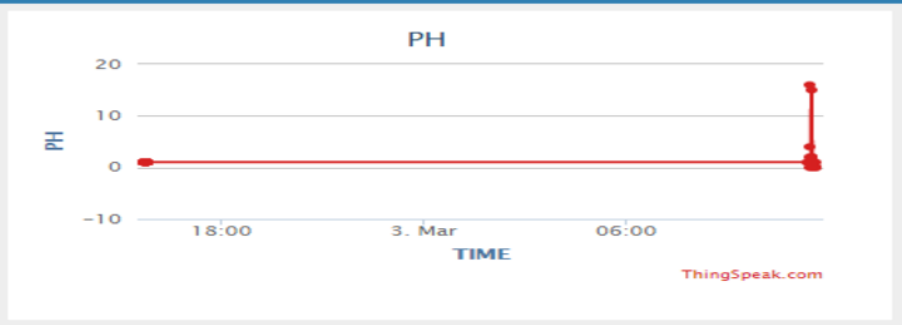

Figure 6. pH sensor 


\section{Future Enhancement}

Air and water quality monitoring has always been under common implementation. Contamination event detection in air and water are easily implemented with the help of low cost sensors. The concept of detecting warm bodies inside drains has not yet been implemented; hence it can be enhanced with the help of a GPS module to get the location of that particular drain. Since large scale deployment of this project requires accurate information about the location of the sensors and the extent to which the warm body is endangered. With the help of gas sensors the concentration of methane inside the drains can also be obtained to calculate the extent to which the warm body inside the drain could survive.

This project makes use of three different modules such as toxic gas detection, water contamination level monitoring and warm body detection inside drains each of which can be implemented over large scale deployment. This paper only focuses on two main toxic gases such as methane and LPG which can be further extended to detect carbon-di-oxide, natural gas, etc. This paper checks water contamination level using $\mathrm{pH}$ and turbidity sensor which can be further enhanced by using sensors to detect TDS, electrical conductivity and aqua sensors to detect micro organisms inside water to identify various disease causing pathogens.

\section{References}

[1] D. Kohl, Function and applications of gas sensors. J. Phys. D. Appl.Phys., vol. 34, no. 19, pp. R125R149, Oct. 2001

[2] Nikhil Kedia, Water Quality Monitoring for Rural Areas- A Sensor Cloud Based Economical Project, in 1st International Conference on Next Generation Computing Technologies (NGCT-2015) Dehradun, India, 4-5 September 2015. 978-1-4673-6809-4/15/\$31.00 C2015 IEEE .

[3] T. P. Lambrou, C. C. Anastasiou, and C. G. Panayiotou, A nephelometricturbidity system for monitoring residential drinking water quality.in Sensor Networks Applications, xperimentation and Logistics.New York, NY, USA: Springer-Verlag, 2009, pp. 43-55.

[4] ] T. P. Lambrou, C. G. Panayiotou, and C. C. Anastasiou, A low-costsystem for real time monitoring and assessment of potable water qualityat consumer sites. in Proc. IEEE Sensors, Oct. 2012, pp. 1-4.

[5] [5] S. Zhuiykov, .Solid-state sensors monitoring parameters of water qualityfor the next generation of wireless sensor networks.Sens. Actuators B,Chem., vol. 161, no. 1, pp. 1-20, 2012.

[6] A. Aisopou, I. Stoianov, and N. Graham, In-pipe water quality monitoringin water supply systems under steady and unsteady state flowconditions: A quantitative assessment. Water Res., vol. 46, no. 1,pp. 235-246, 2012.

[7] S. Panguluri, G. Meiners, J. Hall, and J. G. Szabo, Distribution systemwater quality monitoring: Sensor technology evaluation methodologyand results. U.S. Environ. Protection Agency, Washington, DC, USA,Tech. Rep. EPA/600/R-09/076, 2009.

[8] 8. Kyoung Nam Ha; Kyung Chang Lee; Suk Lee, Development of PIRsensor based indoor location detection system for smart home. SICEICASE,2006. International Joint Conference, vol., no., pp.2162,2167,18-21 Oct. 2006.

[9] Moghavvemi, M.; Lu Chin Seng, "Pyroelectric infrared sensor forintruder detection," TENCON 2004. 2004 IEEE Region 10 Conference, vol.D, no., pp.656,659 Vol. 4, 21-24 Nov 2004.

[10] 10.http://www.nodemcu.com/index_en.html

[11]LM35 Precision Centigrade Temperature Sensors datasheet

[12] MQ-6 Semiconductor Sensor for LPG datasheet

[13] Design of a smart gas detection system in areas of natural gas Storageana M. C. Ilie, Carmela Vaccaro

[14] http://www.businessdictionary.com/definition/PH-scale.html

[15] http://sensorembedded.com/index.php?main_page=product_info\&products_id=564

[16] Guidelines for Drinking-Water Quality, World Health Organization, Geneva, Switzerland, 2011.

[17] https://www.theseus.fi/bitstream/handle/10024/129380/Sigdel_Bishal_WQMS_Thesis.pdf?sequence=1 
[18]Detecting Direction of Movement Using Pyroelectric Infrared Sensors Jaeseok Yun, Member, IEEE, and Min-Hwan Song.

[19] https://learn.adafruit.com/pir-passive-infrared-proximity-motion-sensor?view=all

[20] M. Shankar, J. B. Burchett, Q. Hao, B. D. Guenther, and D. J. Brady,"Human-tracking systems using pyroelectric infrared detectors," Opt.Eng., vol. 45, no. 10, pp. 106401-1-106401-10, Oct. 2006.

[21] https://thingspeak.com/

[22] Movement Direction and Distance Classification Using a Single PIR Sensor Hirenkumar Gami* Department of Engineering Technology, Miami University, Middletown, OH 45042 USA Member, IEEE.

[23] V.D.Ambeth Kumar (2018), novel wireless sensing system for the welfare of sewer laborers.Healthcare Technology Letters (IET) Volume 5, Issue 4, p. $107-112$

[24] V.D.Ambeth Kumar, G.Saranya , D.Elangovan, V.RahulChiranjeevi, V.D.Ashok Kumar, "IOT Based Smart Museum Using Wearable Device.Lecture Notes in Networks and Systems, Vol.55, pp: 33-42, 2018

[25] V.D.Ambeth Kumar, (2016) Human Life Protection In Trenches Using Gas Detection System.for the Journal of Biomedical Research. Vol.27 (2): 475-484.

[26] V.D.Ambeth Kumar, (2016) .Human security from death defying gases using an intelligent sensor system. for the Sensing and Bio-Sensing Research - Journal - Elsevier, Vol.7, pp.107-114 